\title{
ELABORAÇAO DE PLANO DE CUIDADOS \\ PARA APLICAÇAO DA SISTEMATIZAÇAO \\ DA ASSISTENCIA DE ENFERMAGEM EM \\ ACOMPANHAMENTO OBSTETRICO
}

DOI: 10.48140/digitaleditora.2021.005.5

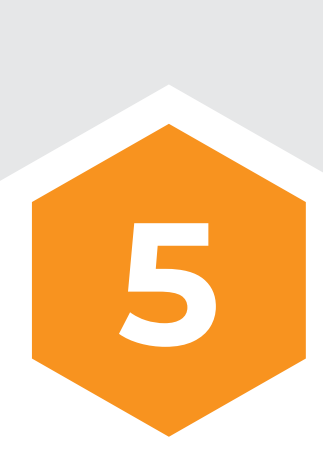

RESUMO

Objetivos: Elaborar um plano de cuidados para Sistematizaçao da Assistência de Enfermagem na obstetrícia.

Métodos: Realizou-se um levantamento bibliográfico nas base de dados SciELO e Bireme num sistema de computação; baseado também no Classificação das Intervenções de Enfermagem (NIC), Classificações dos Resultados de Enfermagem (NOC) e o Diagnóstico de Enfermagem (NANDA) com base nas Necessidades Humanas Básicas mais afetadas no ciclo obstétrico.

Resultados: As Necessidades Humanas mais afetadas na gestação e puerpério foram: sexualidade, conforto, eliminação/troca, nutrição, autopercepção, segurança/proteção e equilíbrio de energia; para tal, foram elencados 12 diagnósticos e 58 intervenções de enfermagem para proposta de elaboração de plano de cuidados.

Conclusão: A assistência humanizada e sistematizada pelo enfermeiro nos períodos clínicos da gestação tem sido um importante elo fazendo com que a gestante aceite as mudanças que acontece nessa fase, o fortalecimento do vinculo, sentimentos e emoções.

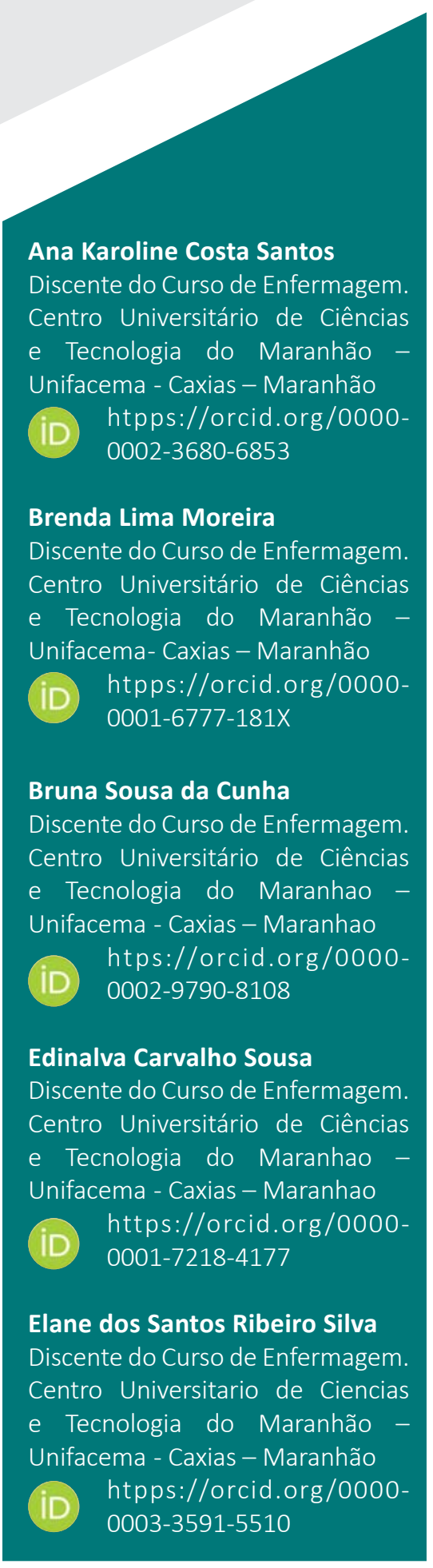

iD Unifacema - Caxias - Maranhao

\section{Brenda Lima Moreira \\ Discente do Curso de Enfermagem. \\ Centro Universitário de Ciências \\ e Tecnologia do Maranhão \\ Unifacema- Caxias - Maranhão \\ htpps://orcid.org/0000- $0001-6777-181 X$ \\ Bruna Sousa da Cunha Discente do Curso de Enfermagem. Centro Universitário de Ciências \\ e Tecnologia do Maranhao \\ Unifacema - Caxias - Maranhao 0002-9790-8108 \\ Edinalva Carvalho Sousa \\ Discente do Curso de Enfermagem. Centro Universitário de Ciências e Tecnologia do Maranhao




\section{PREPARATION OF THE CARE PLAN FOR THE APPLICATION OF THE SYSTEMATIZATION}

OF NURSING ASSISTANCE IN OBSTETRIC

\section{MONITORING}

DOI: 10.48140/digitaleditora.2021.005.5

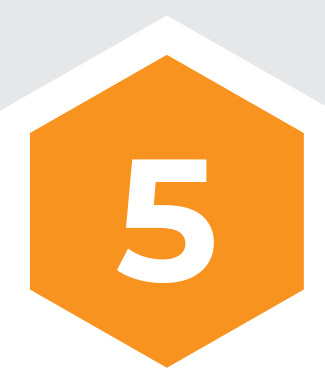

\section{ABSTRACT}

Objectives: Elabore a care plan for Systematization of Nursing Assistence in obstetrics.

Methods: A bibliographic survey was carried out in the SciELO and Bireme databases in a computer system, also based on the Classifications of Nursing Interventions (NIC), Classifications of Nursing Outcomes (NOC) and the Nursing Diagnois (NANDA) based on the Basic Human Needs most affectd in the obstetrics cylcle.

Results: The Human Needs most affectd during pregnancy and the puerperium were: sexuality, comfort, elimination/exchange, nutrition, self-perception, security/protection and energy balance, to this end 12 diagnosis and 58 nursing interventions were listed for the proposal to prepare a care plan

Conclusion: The humanized and systematized assistance by nurses in clinical periods of pregnancy has been an important link making the pregnant woman accept the changes that happen in this phase, the strengthening of the bond, feelings and emotions.

Recebido em: 30/11/2020

Aprovado em: 10/12/2020

Conflito de Interesse: não

Suporte Financeiro: não houve 


\section{INTRODUÇÃO}

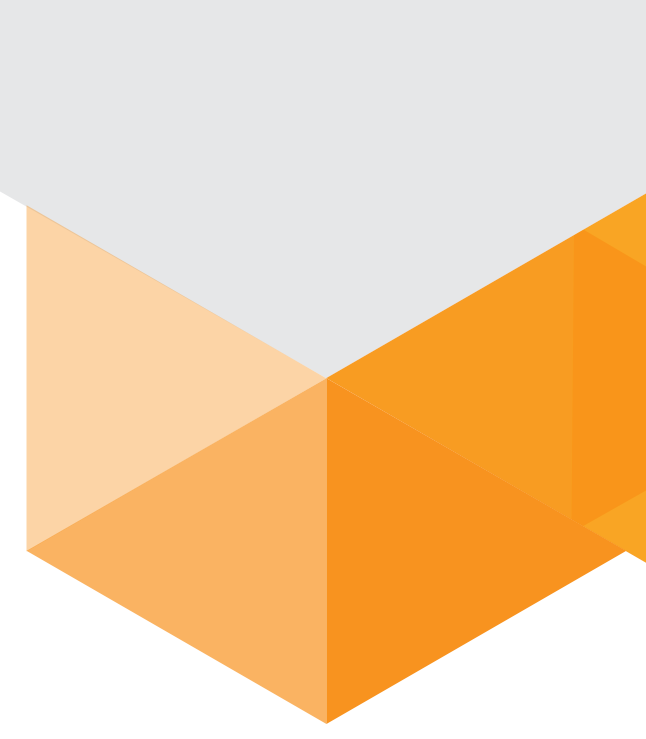

O enfermeiro como integrante da equipe de saúde, assiste a mulher de forma integral. A lei 7498/86 garante o exercício legal da assistência atenção pré-natal, dentre as ações podemos citar a consulta de enfermagem, solicitação de exames de rotina e complementares, prescrições de medicamentos estabelecidos em programas de saúde pública e aprovados pela instituição de saúde, abertura do Sistema de Informações de Saúde (SIS), realização de exames obstétricos, encaminhamentos necessários, preparo para o parto, orientações sobre o cuidados com o recémnascido, amamentação e vacinação (BRASIL, 2002).

O enfermeiro tem competência técnica legal para auxiliar a mulher no período gestacional no que se refere à detecção precoce de intercorrências, diminuir duvidas da gestante e da família, de maneira a contribuir na ampliação da qualidade da assistência na gestação. Desse modo o enfermeiro contribui tanto na gestação de baixo risco quanto no alto risco (DUARTE; ALMEIDA, 2014).

No Brasil, a morbimortalidade materna e perinatal continua altas, sendo que a maioria das mortes e complicações surgem durante a gravidez, o parto e puerpério. Dados do Ministério da Saúde em 2015 indicaram um total de 53.523 mortes maternas, conforme dados do Sistema de Informações sobre Mortalidade (SIM, 2015). Alguns países em desenvolvimento conseguiram melhorar os indicadores com as ações organizadas e integradas. De acordo com o Ministério da Saúde, dentre as complicações destacam-se síndromes hipertensivas, hemorrágicas, descolamento prévio de placenta, desvio do crescimento fetal, macrossomia e outras (BRASIL, 2012).

Segundo o Ministério da Saúde (BRASIL, 2012), a assistência pré-natal, deve ser organizada para atender às reais necessidades da população de gestantes, mediante a utilização dos conhecimentos técnico-científicos existentes e dos meios e recursos disponíveis mais adequados para cada caso. Aa ações de saude devem estar voltadas para a cobertura de toda a população alvo da área de abrangência da unidade de saúde, assegurando minimamente 6(seis) consultas de pré-natal e continuidade no atendimento, no acompanhamento e na avaliação do impacto destas ações sobre a saúde maternal e perinatal.

De acordo com o Decreto no 94.406, de 08 de junho de 1987, e dispõe sobre o exercício da Enfermagem, é privativo do enfermeiro, entre outros, fazer consulta de enfermagem e prescrição assistencial; e como integrantes da equipe de saúde, prescrição de medicamentos previamente estabelecidos 
em programas de saúde pública e em rotina aprovada pela instituição de saúde, assim como prestação de assistência de enfermagem à gestante, parturiente, puérpera e ao recém-nascido.

A Sistematização da Assistência de Enfermagem (SAE) é regulamentada no Brasil pela Resolução no 358/2009 do Conselho Federal de Enfermagem (COFEN), preconizado que sua implantação deva ocorrer em todas as unidades de atendimento à saúde que ofereçam assistência de enfermagem. Esta ferramenta sistemática que orienta e gerencia o cuidado de enfermagem é composta de cinco etapas sequenciais não lineares: histórico de enfermagem ou coleta de dados; diagnóstico de enfermagem; planejamento de enfermagem; implementação da assistência e avaliação de enfermagem.

A utilização desses instrumentos garante ao enfermeiro identificar as necessidades de cada paciente/grupo, direcionado o atendimento a partir de prioridades estabelecidas, o que favorece a implementação de cuidados holísticos, integrais e personalizados.

O processo de enfermagem, se efetivamente praticado proporciona a possibilidade plena de o enfermeiro avaliar a qualidade da assistência prestada, justificando a enfermagem como uma ciência pela aplicação de conceitos e teorias próprias fundamentadas nas ciências biológicas, comportamentais e humanas sempre presentes no processo de cuidar (FANTAUZZI, 2017).

É importante ressaltar e sistematizar a assistência a uma paciente com gestante, de modo que produza resultados que possam ser mensurados, permitindo ampliação dos conhecimentos e melhora na qualidade assistência e do registro das informações de enfermagem.

O objetivo elaborar um plano de cuidado para a sistematização da assistência de enfermagem em obstetrícia.

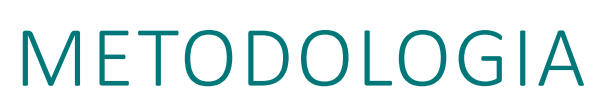

Trata-se de uma pesquisa descritiva, bibliográfica, do tipo revisão da literatura com abordagem qualitativa na qual tem a finalidade e reunir e sintetizar os resultados encontrados sobre uma determinada fase da mulher, de maneira sistematizada para melhor compreensão e aplicação do processo de enfermagem.

A pesquisa qualitativa é uma atividade sistematizada, orientada a compreensão em profundidade de fenômenos educativos e sociais, a transformação de práticas e cenário socioeducativos, a tomada de decisões e também ao descobrimento e desenvolvimento de um corpo organizado de conhecimento (ESTEBAN, 2010, p.127).

Segundo Marconi e Lakatos (2010) explicam que a abordagem qualitativa se trata de uma pesquisa que tem como premissa, análise e interpretar aspectos mais profundos, descrevendo a complexidade de comportamento humano e ainda fornecendo análise mais detalhada sobre as investigações, atitudes e tendência de comportamento.

O foco da pesquisa qualitativa demanda compreender e aprofundar o conhecimento sobre os fenomenos desde a percepção dos participantes ante um context natural e relacional da realidade que os rodeia, com base em suas experiências, opniões e significados, de modo a exprimir suas subjetividades. (MINAYO, 2014).

Segundo a resolução do COFEN 358/2009, o processo de enfermagem organiza-se em cinco etapas interrelacionadas, interdependentes e recorrentes:

A etapa da coleta de dados é a coleta deliberada e sistematizada 
de dados para determinar o estado de saúde e estado funcional atual e anterior do cliente e para determinar os padrões de ajustes atuais e anteriores do cliente.

O diagnóstico de enfermagem é um julgamento clinico a respeito de respostas de indivíduos família ou comunidade a problemas de saúde ou processos vitais reais ou potenciais.

O planejamento é estabelecimento de prioridades e a ordenação dos diagnósticos de enfermagem ou problemas do cliente usando noções de urgência e ou importância para estabelecer uma ordem preferencial para as ações de enfermagem.

Implementação de uma intervenção de enfermagem é qualquer tratamento, baseado em critérios clínicos e no conhecimento, que uma enfermeira realiza para melhorar as condições do cliente.

A avaliação é uma etapa final do processo de enfermagem, e crucial para determinar se a condição ou bem-estar do cliente melhorou após a aplicação do processo de enfermagem.

\section{RESULTADOS E DISCUSSÃO}

Um dos focos em alta, são as atividades de enfermagem em nível ambulatorial, sendo a consulta de enfermagem, onde o profissional de Enfermagem desempenha um papel importante e essencial junto aos usuários, num sentido mais amplo, tendo como objetivo, repassando uma assistência integral, a favor de uma melhoria do estado no que se diz a saúde do assistido, no caso a gestante.

É importante observarmos o quanto se faz necessário estes profissionais, analisando um passo significativo da enfermagem, tanto na função assistencial quanto nas funções gerenciais, dentro dos padrões de atribuições da Enfermagem nos setores ambulatoriais.

Vale ressaltar que a consulta de enfermagem à gestante, baseia-se no Processo de Enfermagem como forma de sistematizar os procedimentos de intervenção de Enfermagem a partir dos problemas identificados na cliente. A importância da aplicabilidade do Processo na Consulta de Enfermagem pode: propiciar uma visão global das condições de saúde da gestante e das ações de enfermagem; Individualizar a assistência prestada; Possibilitar o acompanhamento adequado da evolução das condições da gravidez por parte do profissional; Fornecer dados para futuras pesquisas na área Materno-infantil.

\section{HISTÓRICO DE ENFERMAGEM}

A história clínica objetiva situar a mulher no universo do período gravídicopuerperal, procurando selecionar entidades e situações que possam, de alguma forma, comprometer a evolução da gestação.

A investigação, é a etapa inicial do processo de enfermagem, é a coleta sistemática de informações ou dados. Ela inicia com o primeiro contato do enfermeiro com a paciente e continua tanto tempo quanto existir em necessidade de cuidados com a saúde. Durante a investigação, um enfermeiro coleta informações para determinar quais são os locais de funcionamento anormal os fatores de risco que contribuem para o problema de saúde e os pontos fortes do paciente (ALFARO-LEFEVRE, 2002).

A gravidez e o parto são fatores marcantes na vida de uma gestante, onde se tem várias emoções e acontecimento, a chegada de uma criança na vida dessa gestante é um momento muito importante e marcante, mas sempre traz alguns impactos positivos e negativos relacionados a maneira, o convívio 
de que forma são tradadas, fazendo com que se tronem inseguras e frágeis devido ao fato de seu bebê ter algum problema de saúde ou alguma anomalia (CUNHA, 2015)

\section{Característica Do Atendimento Á Gestante}

Especificamente, são quarto as atividades básicas que no enfermeiro deve realizar na consulta à gestante no momento da coleta de dados ou Histórico de Enfermagem:

1. Entrevista;

2. Exame Físico;

3. Exame Obstétrico;

4. Orientação.

\section{ENTREVISTA}

Compreende-se como entrevista a coleta de dados da paciente com fins a investigar:

- Identificação da gestante: idade; endereço e nome da gestante, religião, profissão; estado civil.

- Antecedentes pessoal e familiar: historia de doenças anteriores; diabetes; hipertensão; cancer; doenças congenita; gemelaridade; cardiopatia e doenças renais crônica; anemia; transfusão de sangue.

- Condições socioeconômicas e culturais: grau de instrução, número de pessoas dependentes; renda familiar per capita; pessoas da familia que participam da força de trabalho; condiçoes de moradias.

- Situações relacionadas com o estado gestacional (história gestacional e paridade): número de gestações; número de partos; número de abortamentos; número de filhos vivos; idade da primeiras gestação, intervalos entre as gestações; natimortos; recém-nascido com íctericia; intercorrência ou complicações em gestações anteriores; complicações no puerpérios; historia de aleitamento anteriores e intervalo entre o final da ultima gestação e o início da atual.

- Condições de saúde atual: data da primeiro dia/mês/ano da ultima menstruação DUM; data provável do parto DPP; data da percepção dos primeiras movimentos fetais; sinais e sintomas na gestação em curso; medicamentos usados na gestação; a gestação foi ou não desejada; hábitos (fumo, álcool e drogas ilícitas) e ocupação habitual.

EXAME FISICO: Faz-se necessário a avaliação da gestante através do exame físico a partir dos passos a seguir:

- Verificação do peso e altura;

- Determinação da frequencia cardíaca;

- Inspeção da mama;

- Condições dos dentes;

- Existência de edema e varizes;

- Temperature axillar;

- Aferição da pressão arterial;

- Inspeção da pele e mucosas;

- Palpação da tireóide;

- Ausculta cardiopulmonar;

- Exame do abdomen; 
- Palpação dos gânglios inguinais;

- Exame dos membros inferiores;

- Pesquisa de edema.

\section{EXAME OBSTÉTRICO}

A partir do estágio da gestação, é imprescindível, ser realizadas as ações abaixo:

- Medida da circunferência abdominal;

- Fundo do útero;

- Apresentação;

- Situação;

- Posição;

- Batimentos cardiofetal-BCF

Para tal, elaborou-se um modelo de Histórico para coleta de dados obstétricos, conforme quadro abaixo 1 abaixo.

Quadro 1: Proposta de Histórico de Enfermagem para coleta de dados obstétricos. Caxias-2021.

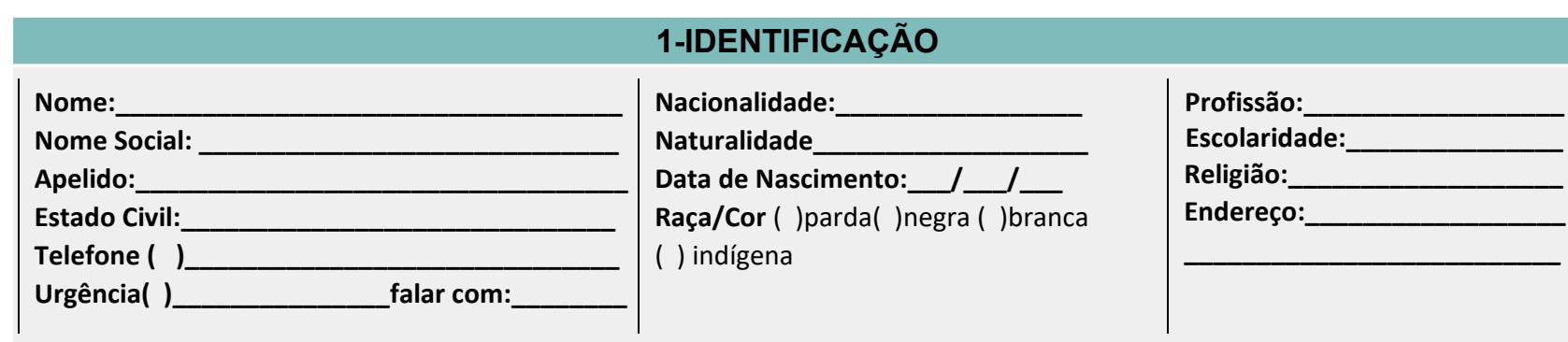

\section{2- ANTECEDENTES PESSOAIS}

\begin{tabular}{|c|c|c|c|c|}
\hline $\begin{array}{l}\text { Peso: } \\
\text { IMC: }\end{array}$ & Altura: ___ cm & \multicolumn{2}{|c|}{ IMC: >25 obeso; <20 baixo peso } & PA:___sistólica____diastólica \\
\hline $\begin{array}{l}\text { Etilismo: ( )SIM ( )NÃO } \\
\text { Quantidade/dia: } \\
\text { Tempo de consumo: }\end{array}$ & $\begin{array}{l}\text { Tabagismo: ( )SIM } \\
\text { ( )NÃO ( )PAROU } \\
\text { Quant./dia: } \\
\text { Tempo: }\end{array}$ & \multirow{2}{*}{\multicolumn{2}{|c|}{$\begin{array}{l}\text { Drogadição: ( )SIM( )NÃO( )PAROU } \\
\text { Qual: } \\
\text { Forma de uso: } \\
\text { Tempo de consumo: } \\
\text { Parou há quanto tempo? }\end{array}$}} & \multirow[t]{2}{*}{$\begin{array}{l}\text { Atividade Física: ( )SIM( )NÃO } \\
\text { Frequência: } \\
\text { Sedentário? ( )SIM( )NÃO }\end{array}$} \\
\hline 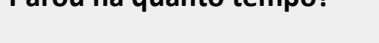 & rempo que parou: & & & \\
\hline № de gestações: & Paridade: & $\begin{array}{l}\text { Abortamentos: } \\
\text { ( )SIM( )NÃO } \\
\text { Quantos: }\end{array}$ & $\begin{array}{l}\text { Curetagem: } \\
\text { ( )SIM( } \\
\text { )NÃO }\end{array}$ & $\begin{array}{l}\text { Parto: } \\
\text { Normal ( ) Quantidade: } \\
\text { Cesáreo ( ) Quantidade: }\end{array}$ \\
\hline $\begin{array}{l}\text { Evacuações presentes: ( )SIN } \\
\text { Normal ( ) } \\
\text { Ressecada ( ) } \\
\text { Pastosa ( ) } \\
\text { Ausente ( ) }\end{array}$ & 1（） NÃO & \multicolumn{3}{|c|}{$\begin{array}{l}\text { Diurese ( )SIM( )NÃO } \\
\text { Cor: ( )Amarelo claro( )Amarelo( ) Âmbar( )Marrom ( ) Vermelho } \\
\text { Odor:( )SIM NÃO( ) }\end{array}$} \\
\hline \multicolumn{2}{|c|}{ Imunização: Atualizada ( ) Em atraso ( ) } & \multicolumn{2}{|c|}{$\begin{array}{l}\text { Cirurgias prévias:( ) SIM NÃO（） } \\
\text { Quais? }\end{array}$} & $\begin{array}{l}\text { lergias: :( ISIM NÃO（） } \\
\text { uais? }\end{array}$ \\
\hline
\end{tabular}

\section{3-HISTÓRICO DE PATOLOGIAS}

( ) Diabetes ( )Hipertensão Arterial ( )Cardiopatas ( ) Vasculopatias ( )DPOC ( )Doença Renal ( ) Câncer de mama ( )Câncer Ginecológico ( )Infecção Urinária ( )Outras Medicações de uso continuo: ( ) SIM( )NÃO Dose: Via: Horário:

\section{( )Uso de cosméticos}

$$
\text { Quais? }
$$

\section{4-ANTECEDENTES FAMILIARES}

( )Diabetes ( )Hipertensão Arterial ( ) Cardiopatias ( ) Gemelidade ( )Câncer de mama ( )Câncer Ginecológico ( ) Malformações ( ) Outras 
5-ANTECEDENTES GINECOLÓGICOS

\begin{tabular}{|c|c|}
\hline Data da última menstruação: _________________ & Duração do ciclo: \\
\hline \multicolumn{2}{|c|}{ Duração do fluxo menstrual:_____dias Fluxo: Leve ( ) Médio( ) Intenso ( ) Sangramento entre os ciclos ( )SIM ( )NÃO } \\
\hline Idade da menopausa: _______ Tratamento hormonal: ( ) SIM ( )NÃO & Sangramento durante as relações: ( )SIM ( )NÃO \\
\hline Método anticoncepcional: ( )SIM ( )NÃO № de parceiros no último ano:_ & _História de ISTs: ( )SIM ( )NÃO Quais? \\
\hline
\end{tabular}

\title{
6- ANTECEDENTES OBSTÉTRICOS
}

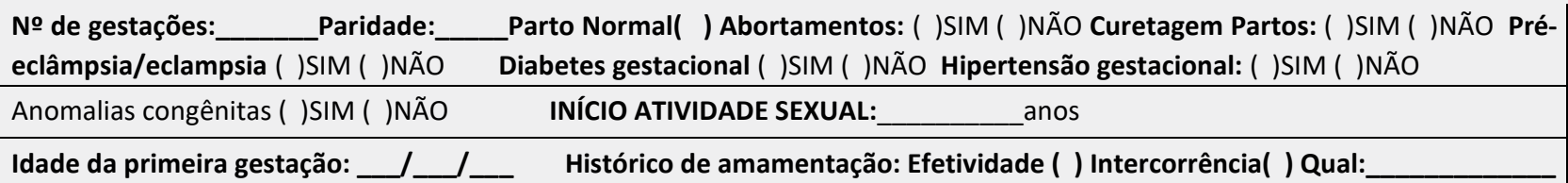

\section{7-EXAME FÍSICO}

\author{
SINAIS VITAIS: \\ PA: \\ P: \\ ( )Filiforme ( )Cheio ( ) Rítmico ( ) Arrítmico \\ $\mathrm{T:}$ \\ Oxigênio: \\ FR: \\ Nível de Consciência: \\ Dor: ( )SIM ( )NÃO \\ Onde?
}

()Consciente ()Orientado ()Desorientada

Aspecto Emocional: ( )Calma ( ) Tensa ( )Agitada ( )Apática ( ) Eufórico ( ) Agressiva ( )Triste

\section{CABEÇA}

Couro cabeludo: ( ) sem anormalidades ( )ferimento ( )hematomas ( )seborreia ( )parasitos

Face: () simétrica ( )assimétrica ( )edema ()tipos de fáceis

Olhos: ( )normal ( )baixa acuidade visual ( )lentes ( )processos inflamatório/infeccioso ( )exoftalmia/enoftalmia ( )estrabismo

Pupilas: ( )isocóricas ( ) anisocóricas () miose bilateral ( )midríase bilateral

Nariz: ( )simétrico ( ) assimétrico ( )sem anormalidade ( )secreção ( )lesão

Ouvidos: ( ) deformações congênitas ou adquirida ( )otorreia ( )otorragia

Boca: ( )sem anormalidade ( )cárie ( )falhas dentárias ( )prótese

Pescoço: ( )simetria ( )veias jugulares ( )glândulas parótidas

\section{SISTEMA RESPIRATÓRIO}

Padrão: ( )eupnéico ( )bradipnéico ( )taquipnéico ( )dispneico ( )tiragem intercostal

Inspeção pulmonar: ( )normal ( )diminuída ( )simetria ( )assimetria

Palpação: ( )traquéia ( )expansibilidade ( )frêmitos

Ausculta: murmúrio vesicular ( )presente ( )diminuído ( )ausente

Ruídos adventícios: ( )roncos ( )sibilos ( )estertores ( )tosse

Alterações:

\section{SISTEMA CARDIOVASCULAR}

Perfusão periférica: ( )normal ( )diminuída ( )cianose outros:

Ausculta cardíaca: ( )normal ( )bradicardia ( )taquicardia ( )ritmo regular ( )irregular

\section{SISTEMA GASTROINTESTINAL}

Abdômen: ( )Normal ( )globoso ( ) ventre de batráquio ( ) avental ( ) pendular ( ) escavado ( ) doloroso Ruídos hidroaéreos: ( )ausente ( )presente, TIPO: ( )hiperativos ( )hipoativos Dieta: via:

Eliminação Intestinal: ( )normal ( )constipação ( )diarreia ( )melena

\section{EXAME FISICO GINEO-OBSTÉTRICO}

Inspeção: ( )Coloração ( )cicatrizes

Palpação: Manobras de Leopold

Situação fetal: ( )longitudinal ( )transversal

Apresentação: ( )cefálica( )pélvica

Mensuração: CRESCIMENTO ( ) adequado ( )inadequado

Ausculta: BCF ( )presente ( )ausente 


\section{EXAMES COMPLEMENTARES SOLICITADOS À GESTANTE}

Quadro 2: Exames complementares solicitados durante período gestacional. Caxias-MA, 2021.

\section{PRIMEIRO TRIMESTRE}

Tipagem sanguínea $(\mathrm{ABO} / \mathrm{Rh})$

Teste de Coombs indireto (se indicado).

Hemograma completo.

Glicemia de jejum.

Urina tipo 1

Urocultura com antibiograma

SOROLOGIA:

Rubéola

Toxoplasmose

Sífilis

Citomegalovírus

Hepatite B e C

HIV

$\mathrm{TSH}$

Parasitológico de fezes (PPF).

Citologia oncótica

Ultrassonografia morfológica de primeiro trimestre com perfil bioquímico.

\section{SEGUNDO TRIMESTRE}

TERCEIRO TRIMESTRE

Hemograma completo

Glicemia em jejum

Hemograma completo

Teste de tolerância oral à glicose

(TTOG) de $75 \mathrm{~g}$;

\section{SOROLOGIAS}

Sífilis

Toxoplasmose

SOROLOGIAS Toxoplasmose

Hepatite B

Sífilis.

HIV

Urina tipo 1

\section{EXAMES DE IMAGEM}

Ultrassonografia morfológica de 20 trimestre com Dopplerfluxometrica colorida das artérias uterinas maternas e avaliação do colo por via vaginal.
Urocultura com antibiograma Cultura seletiva para estreptococos hemolíticos de introito vaginal e perianal.

\section{EXAMES DE IMAGEM}

Ultrassonografia obstétrica com Dopplerfluxometrica colorida; Ecocardiografia fetal.

Cardiotocografia basal.

\section{DIAGNÓSTICO DE ENFERMAGEM}

É o julgamento clinico das respostas do indivíduo, da família ou da comunidade a problemas de saúde/processo vitais, reais ou potenciais (NANDA, 2013). Os diagnósticos de enfermagem serão apresentados em forma de quadro juntamente com a terceira etapa do processo de enfermagem.

\section{PLANEJAMENTO}

O planejamento ou processo priorização dos diagnósticos de enfermagem e dos problemas colaborativos, identificando as metas mensuráveis ou resultados selecionados intervenções adequadas e 
documentando o plano de cuidado (POTTER, 2013).

Quadro 3: Distribuição dos Diagnósticos de Enfermagem (NANDA), Resultados Esperados (NOC) e Intervenções de Enfermagem (NIC) com base nas Necessidades Humanas Básicas da gestante no ciclo gravídico peuerperal. Caxias-MA, 2021

\begin{tabular}{|c|c|c|c|c|c|}
\hline \multicolumn{6}{|c|}{ NECESSIDADES PSICOBIOLOGICAS, PSICOSSOCIAIS E RELIGIOSAS } \\
\hline $\begin{array}{l}\text { Necessi- } \\
\text { dades } \\
\text { Humanas } \\
\text { Básicas } \\
\text { afetadas }\end{array}$ & $\begin{array}{l}\text { Diagnósticos de } \\
\text { Enfermagem } \\
\text { (NANDA }\end{array}$ & $\begin{array}{c}\text { Fator } \\
\text { Relacionado }\end{array}$ & $\begin{array}{c}\text { Características } \\
\text { Definidoras }\end{array}$ & $\begin{array}{l}\text { Resultados } \\
\text { Esperados } \\
\text { (NOC) }\end{array}$ & $\begin{array}{l}\text { Intervenções sugeridas } \\
\text { (NIC) }\end{array}$ \\
\hline 岁 & $\begin{array}{l}\text { Padrão de se- } \\
\text { xualidade inefi- } \\
\text { caz }\end{array}$ & $\begin{array}{l}\text { Conheci- } \\
\text { mento insufi- } \\
\text { ciente sobre } \\
\text { a função } \\
\text { sexual }\end{array}$ & $\begin{array}{l}\text { Mudança in- } \\
\text { desejada na } \\
\text { função sexual. } \\
\text { Dificuldades } \\
\text { com atividade } \\
\text { sexual }\end{array}$ & $\begin{array}{l}\text { Conheci- } \\
\text { mento: } \\
\text { Funciona- } \\
\text { mento se- } \\
\text { xual na } \\
\text { gravidez e } \\
\text { no pós-parto }\end{array}$ & $\begin{array}{l}\text { Promover a responsa- } \\
\text { bilidade comportamen- } \\
\text { to sexual; -Discutir os } \\
\text { benefícios da abstinên- } \\
\text { cia; } \\
\text { - Dizer ao paciente que } \\
\text { você está preparado } \\
\text { para responder as per- } \\
\text { guntas sobre a função } \\
\text { sexual; } \\
\text { Aconselhar a paciente } \\
\text { sobre as mudanças na } \\
\text { sexualidade durante a } \\
\text { gravidez; } \\
\text { Dar informações con- } \\
\text { cretas sobre mitos e } \\
\text { informações erradas } \\
\text { sobre sexo que o pa- } \\
\text { ciente possa expres- } \\
\text { sar. }\end{array}$ \\
\hline 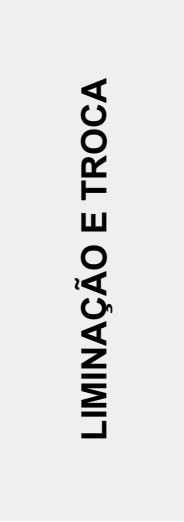 & Constipação & $\begin{array}{l}\text { Hábitos } \\
\text { de eva- } \\
\text { cuação } \\
\text { irregula- } \\
\text { res; } \\
\text { - Ingestão } \\
\text { de fibras } \\
\text { insufi- } \\
\text { ciente; }\end{array}$ & $\begin{array}{l}\text { Esforço para } \\
\text { evacuar; Fezes } \\
\text { formadas endu- } \\
\text { recidas; }\end{array}$ & $\begin{array}{l}\text { Eliminação } \\
\text { intestinal }\end{array}$ & 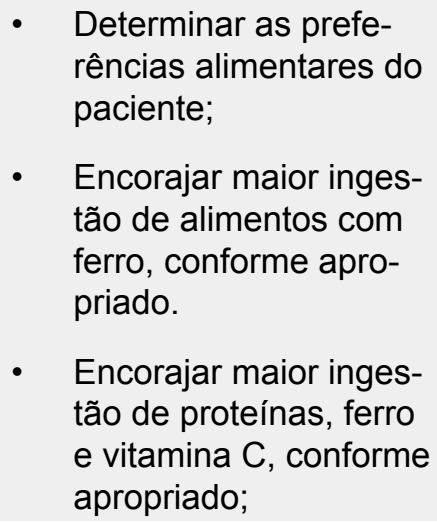 \\
\hline
\end{tabular}




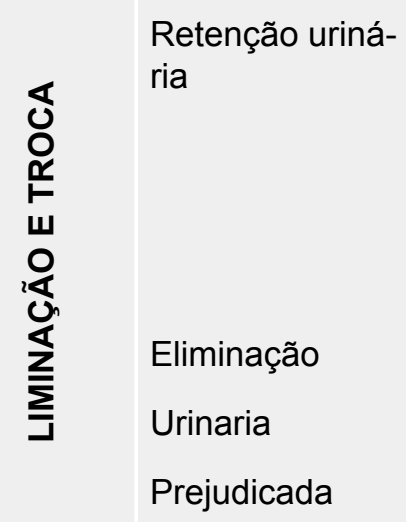

通

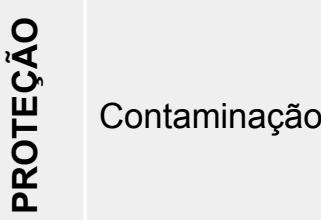

㟔

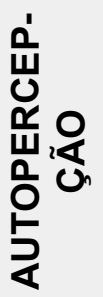

Urinar com

frequência Por gravidez

Multiplas

Causas

Idade ges-

tacional du-

rante

exposição;

gravidez

Gravidez

Distúrbio de

Relatar incapacidade de manter rotinas habituais

Retenção urinaria

Agentes biológicos
Conhe-

cimento controle de infecção

Eliminação
Urinária

Comportamento

de Saúde Prénatal

Estratégias para equilibrar atividade $\mathrm{e}$ repouso

Nível de imagem cor- confiança autoestima poral

- Usar técnicas para eliminação urinária; -Proporcionar privacidade para eliminação; -Oferecer tempo suficiente para o esvaziamento da bexiga (10 minutos).

Monitorar a vulnerabilidade a infecção;

Orientar a paciente aos cuidados com a higiene intima;

Orientar paciente quanto ao uso de antibiótico conforme prescrição médica;

Monitorar a ingetão e a eliminação;

Usar tecnica para a eliminação urinária.

- Garantir segurança da água

- instituindo, hipercloração e hiperaquecimento conforme apropriado; -Monitorar as mudanças no nível de energia/malestar;

- Estimular repouso;

- Promover;

- Administrar agente imunizante conforme apropriado.

- Escolher um cenário calmo e confortável;

- Orientar a paciente a respirar profundamente e a lentamente, expirar e aliviar a tensão; -Concluir, pouco a pouco, a sessão de relaxamento.

- Evitar críticas negativas; - Encorajar o paciente a identificar os pontos fortes;

- Transmitir confiança na capacidade do paciente para lidar com a situação. 


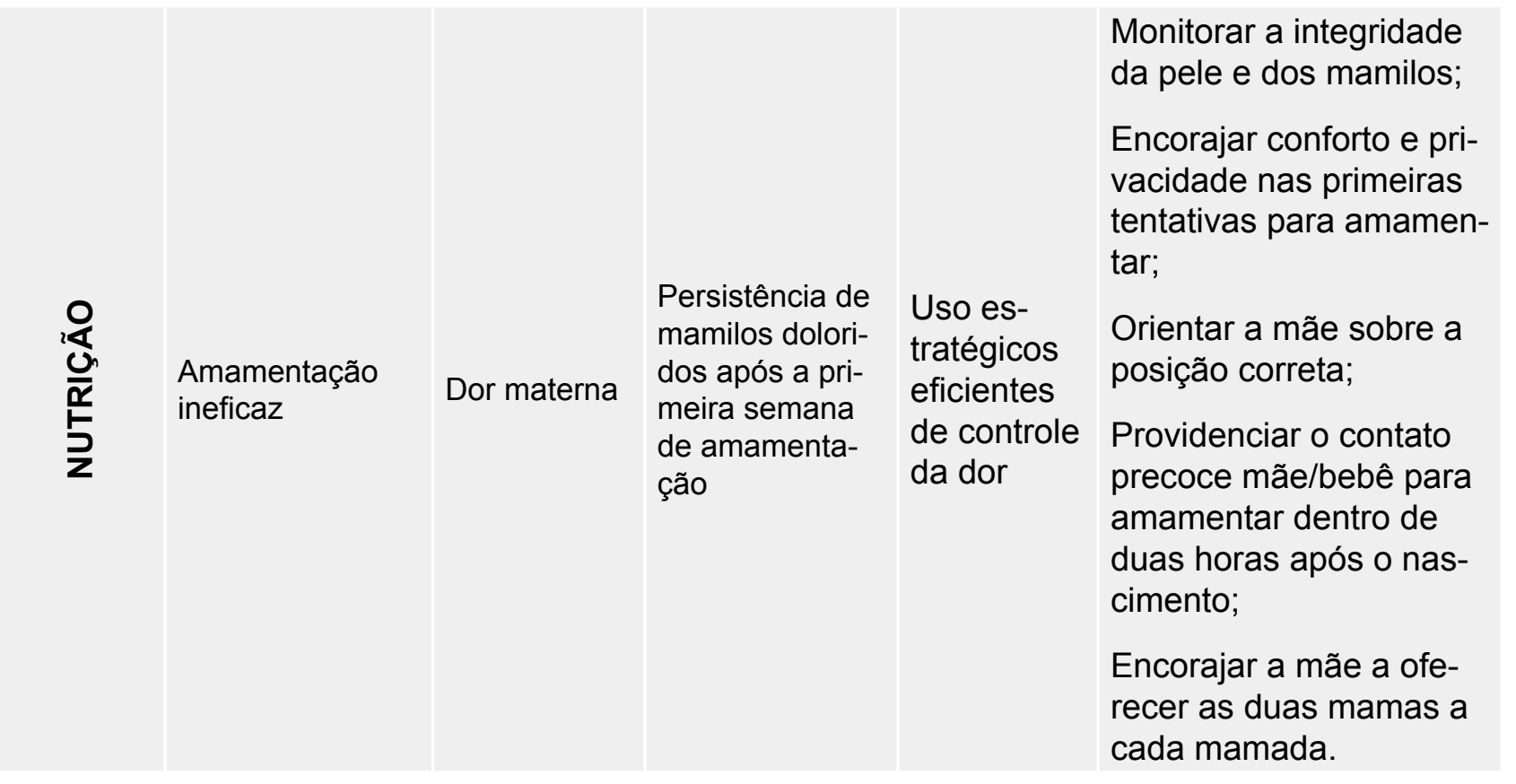

\section{IMPLEMENTAÇÃO}

A quarta etapa do processo de enfermagem, começa formalmente depois que a enfermeira desenvolve um plano de tratamento baseado na realização da ações em interveções de enfermagem de forma clara e relevante (POTTER, 2013).

- Assegurar que a dieta inclua alimentos ricos em fibras;

- Promover sono e repouso adequado para facilitar o alivio das náuseas;

- Promover responsabilidade comportamento sexual;

- Monitorar a paciente em relação a comportamentos relativos a atos alimentares, perda e aumento de peso;

- Remover dos ambientes objetos potencialmente perigosos;

- Escolher um ambiente calmo e confortável.

\section{AVALIAÇÃO}

O passo final do processo de enfermagem, é crucial para determinar se, após a aplicação do processo de enfermagem, a condição do cliente ou seu bem-estar melhoraram (POTTER, 2013). 


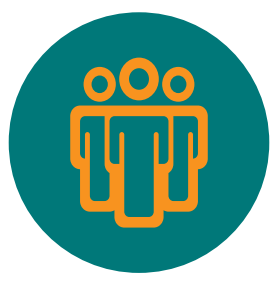

\section{CONCLUSÃO}

A atenção adequada no momento do parto representa uma relação em que a mulher e a familia estabelecerão com a criança desde as primeiras horas do nascimento, fortalecer vinculo, sentimentos, emoções percebidos e o fato importante de que uma gestante precisa é ser escutada onde se sentirá acolhida e poderá expressar suas dificuldades, um passo indispensável para garantir que ela possa vivenciar a experiência da maternidade com segurança e bem-estar. Este é um direito fundamental de toda mulher.

A equipe de enfermagem em obstetrícia deve estar preparada para acolher a gravida, seu companheiro e familiar, respeitando todos os significados desse momento. Isso deve facilitar a criação de vinculo mais profundo da equipe coma gestante, ao Ihe transmitir confiança e tranquilidade, oriundas das estratégias para humanização da atenção durante o acompanhamento no pré-natal, parto e pós-parto institucionalizado. 


\section{REFERÊNCIAS}

ATENÇÃO AO PRÉ-NATAL DE BAIXO RISCO, Série A. Normas e Manuais Técnicos Caderno de Atenção Básica, no 32, Brasilia, 2012.

ARAUJO, Osa Maria Machado de. CONSULTA DE ENFERMAGEM À GESTANTE. Rev. Bras. Enferm., Brasília, v. 32, n. 3, p. 259-270, 1979. Available from <http://www.scielo.br/scielo.php?script=sci_arttext\&pid=S0034-71671979000300259\&Ing=en\&nrm=iso>. access on 19 Mar. 2021. http://dx.doi. org/10.1590/0034-716719790003000005.

BRILHANTE, A. F; VASCONELOS, C. T. M; BEZERRA, R. A; LIMA, S. K. M; CASTRO, C. M. B; FERNANDES, A. F. C, Implementação do protocolo de acolhimento com classificação de risco em uma emergência obstétrica. Ver. Rene, Fortaleza/Ceara, v.17, n. 4, p. 569-75,2016.

BRASIL. Ministério da Saúde. Assistência Pré-Natal Manual Técnico, Brasilia ,2000 Disponivel em:bvsms,saude.gov.br/bvs/publicações/cdo4-11pdf.

ESTEBAN, M. P. S. Pesquisa qualitativa em educação. Porto Alegre: Artmed, 2010.

NANDA. Diagnóstico de enfermagem da NANDA: definições e classificação 2015-2017 / [NANDA International]; organizadoras: t. Heather Herdman, Shigemi Kamitsuru ; tradução : Regina Machado Garcez ; revisão técnica: Alba Lucia Machado Barros...[et al.]. - Porto Alegre : Artmed, 2015.

NOC. Classificação dos resultados de enfermagem (NOC) / Sue Moorhead_[et al,]; [tradução Regina Machado Garcez... et al]. - Rio de Janeiro; Elsevier, 2010. Classificações de intervenções de enfermagem (NIC) / , Howard Gloria m. Bulechek k, Butcher; Joanne McClockey Dochterman ; [tradução Soraya Imon de Oliveira... et al] - Rio de Janeiro : Elservier, 2010.

Enfermeira Global Assistência pré-natal: ações essenciais desenvolvidas pelos enfermeiros. Revista electronica trimestral de Enfermagem, n 40, Octuber 2015, www.um.es/eglobal.

SARNEY, J; ALMEIDA, E. A. COFEN Conselho Federal de Enfermagem, Dec, no 94.406, Brasilia de 08/06/1987, publicado no DOU de 09/06/1987.

Ligações NANDA - NOC - NIC: condições clínicas: suporte ao raciocínio e assistência de qualidade/Marion Jhonson... [ et al.;tradução de Soraya Imon de Oliveira... et al. ] . - Rio de Janeiro: Elsevier, 2012. .

MARCONE, Marina de Andrade; LAKATOS, Eva Maria, Fundamentos de Metodologia Cientifica. 7ed. São Paulo: Atlas, 2010.

MINAYO, M. C. S. O desafio do conhecimento: pesquisa qualitativa em saúde. São Paulo (SP): Hucitec, 2014.

POTTER, Patricia Ann Fundamentos de enfermagem/ Patricia A. Potter, Anne Griffin Perry ;[ tradução de Maria Inês Corrêa Nascimento... et al.]. - Rio de Janeiro : Elsevier, 2011.

Potter, Patricia Ann Fundamentos de enfermagem/ Patricia A. Potter, Anne Griffin Perry ; editores das seções May Hall, Patricia A. Stocker ; [ tradução de Mayza Ritomy Ide ... et al.] . - Rio de Janeiro : Elservier, 2013.

SILVA, M. C. N, Resolução COFEN-358/2009, Brasilia 15 de Outubro, 2009.

TANNURE, Meire Chucre, SAE: Sistematização de Assistencia de Enfermagem: Guia Prático/ Meire Chucre Tannure. Ana Maria Pinheiro, 2. ed, - [Reimper.] - Rio de Janeiro: Guanabara Koogan, 2011. II. Disponivel em < http://www.who.int/mentalhealth/media/68.pdf > . Acesso em outubro de 2019. 